\title{
Reflexiones acerca de autoetnografía interpretativa: un camino para humanizar el cuidado de Enfermería ${ }^{1}$
}

Institución: Universidad de Chile

\author{
Mirliana Ramírez-Pereira ${ }^{2}$ \\ Mónica Ferrada Muñoz ${ }^{3}$ \\ Esmérita Opazo Morales ${ }^{4}$ \\ Silvia Rojas Núñez
}

\section{ENSAYO}

\section{RESUMEN}

Este ensayo expone una reflexión sobre la autoetnografía interpretativa, una metodología poco explorada en la lengua española y en el área de la salud, la cual rescata la sensibilidad de la atención, enmarcada en el yo, la cultura y el método. Como método, los autores de este trabajo utilizan la reflexión acerca de la experiencia en cuanto a la provisión de cuidados de enfermería y la autoetnografía como método, complementada con la revisión y análisis de artículos de revistas y libros. Se concluye que la enfermera puede usar este método si se conoce y se reconoce a sí como un ser humano con múltiples posibilidades y potencialidades, pero también con imperfecciones y limitaciones y con la posibilidad de mirar al otro, siendo consciente de que el paciente es un ser humano tan perfecto e imperfecto como ella.

Palabras claves: atención-dirigida-al-paciente; enfermería; investigación-cualitativa.

DOI: $10.15517 /$ revenf.v0i36.32912

${ }^{1}$ Fecha de recepción: 7 de abril del 2018

${ }^{2}$ Académica. Doctora en Enfermería. Departamento de Enfermería, Universidad de Chile. Chile. Correo electrónico: mirliana@uchile.cl

${ }^{3}$ Académica. Carrera de Enfermería. Departamento de Clínicas. Universidad Católica del Norte. Chile. Correo electrónico: mferrada@ucn.cl

${ }^{4}$ Académica. Departamento de Enfermería. Universidad de Chile. Chile. Correo electrónico: esmeritaopazo@med.uchile.cl

${ }^{5}$ Docente. Departamento de Enfermería. Universidad de Chile. Chile. Correo electrónico: silviarojasnunez@gmail.com 


\title{
Reflections on interpretive autoethnography: a way to humanize Nursing care ${ }^{1}$
}

\author{
Mirliana Ramírez-Pereira ${ }^{2}$ \\ Mónica Ferrada Muñoz ${ }^{3}$ \\ Esmérita Opazo Morales ${ }^{4}$ \\ Silvia Rojas Núñez
}

Institution: University of Chile

\section{ESSAY}

\begin{abstract}
A reflection essay on interpretive autoethnography is presented, a methodology little explored in the Spanish language and health. This methodology rescues the sensitivity of health care, framed in the self, culture and method. We use as a method the reflection about the experience lived by the authors in the provision of nursing care, as well as the experience in autoethnography like a method, complemented with review and analysis of journal articles and books. It is concluded that the nurse can use this method if she knows and recognizes herself as a human being with multiple possibilities and potentialities, but also with imperfections and limitations and with the possibility of looking at the other, being aware that the patient is a human being as perfect and imperfect as her.
\end{abstract}

Key Word: qualitative-research; nursing; patient-centered-care.

DOI: $10.15517 /$ revenf.v0i36.32912

${ }^{1}$ Date of receipt: April 7, 2018

Date of acceptance: October 8, 2018

2 Academic. Doctor's degree in Nursing. Department of Nursing, University of Chile. E-mail: mirliana@uchile.cl

${ }^{3}$ Academic. Career of Nursing. Department of Clinics. Catholic University of the North. E-mail: mferrada@ucn.cl

${ }^{4}$ Academic. Department of Nursing. University of Chile. E-mail: esmeritaopazo@med.uchile.cl

${ }^{5}$ Professor. Department of Nursing. University of Chile. E-mail: silviarojasnunez@gmail.com 


\title{
Reflexões sobre a autoetografia interpretativa: uma forma de humanizar o cuidado de enfermagem ${ }^{1}$
}

\author{
Mirliana Ramírez-Pereira ${ }^{2}$ \\ Mónica Ferrada Muñoz ${ }^{3}$ \\ Esmérita Opazo Morales ${ }^{4}$ \\ Silvia Rojas Núñez
}

Instituição: Universidade do Chile

\section{ENSAIO \\ RESUMO}

Apresentamos um ensaio de reflexão sobre a auto-etnografia interpretativa, uma metodologia pouco explorada na língua espanhola e na saúde. Essa metodologia resgata a sensibilidade da atenção, enquadrada no eu, cultura e método. Utilizamos como método a reflexão sobre a experiência vivida pelos autores na prestação do cuidado de enfermagem, bem como a experiência no uso da auto-etnografia como método, complementada com a revisão e análise de artigos e livros de jornais. Conclui-se que o enfermeiro pode utilizar a autoetnografia interpretativa se é conhecida e reconhecida como um ser humano com múltiplas possibilidades e potencialidades, mas também com imperfeições e limitações e com a possibilidade de olhar para o outro, sabendo que o paciente é tal humano. perfeita e imperfeita como ela.

Palavras-chave: assistência-centrada-no-paciente; enfermagem; pesquisa-qualitativa.

DOI: $10.15517 /$ revenf.v0i36.32912

${ }^{1}$ Data de recepção: 7 de abril de $2018 \quad$ Data de aceitação: 8 de outubro de 2018
${ }^{2}$ Acadêmico. Doutora em Enfermagen. Departamento de Enfermagen. Universidade do Chile. Chile. Correio eletrônico: mirliana@uchile.cl

${ }^{3}$ Acadêmico. Carreira de Enfermagen. Departamento de Clínicas. Universidade Católica do Norte. Chile. Correio eletrônico: mferrada@ucn.cl

${ }^{4}$ Acadêmico. Departamento de Enfermagen. Universidade do Chile. Chile. Correio eletrônico: esmeritaopazo@med.uchile.cl

${ }^{5}$ Professor. Departamento de Enfermagen. Universidade do Chile. Chile. Correio eletrônico: silviarojasnunez@gmail.com 


\section{INTRODUCCIÓN}

Con el fin de construir ciencia, la enfermería ha experimentado diversas transformaciones y ha debido sortear numerosas batallas a lo largo de la historia: en un principio, las enfermeras eran religiosas y más tarde mujeres de dudosa reputación obligadas a cuidar enfermos como castigo por sus delitos y pecados: no fue sino hasta la llegada de Florence Nigthingale que comenzó la enfermería moderna, con los primeros atisbos del uso de la evidencia para la toma de decisiones en salud; por ejemplo, ella indicaba que abrir ventanas, limpiar y dejar entrar la luz eran formas de curación, con un cuidado orientado principalmente al cuerpo físico, el cual persiste hasta nuestros días, disociando al ser humano, comprendiéndolo como una figura dual, en que existe solamente cuerpo y mente, por lo que se olvida aspectos subjetivos y sociales, históricamente dibujados en las distintas culturas ${ }^{1}$.

Tal forma de comprensión del proceso salud- enfermedad ha significado que la enfermería haya basado el cuidado en la ciencia construida por los científicos positivistas, cuyo modelo atiende aspectos físicos, biológicos o fisiológicos, mas descuida los psicosociales, lo que ha llevado a comercializar y deshumanizar la atención de salud de la población ${ }^{2}$.

Al excluir la mirada de las personas, vale decir, su propio sentir, vivencias y contexto, las enfermeras hemos olvidado también elementos económicos, sociales, políticos e históricos, que son fundamentales para comprender el ser humano desde una perspectiva integral, además de olvidar nuestro propio sentir como enfermera-ser humano, que sobrepasa nuestros miedos, angustias y vivencias y que nos convierte en un engranaje más de un sistema que debe ser funcional, hasta homologarnos con máquinas; es decir, no solo hemos deshumanizado el cuidado del otro, sino que también hemos dejado de vernos a nosotras mismas como personas.

En este contexto, presentamos la autoetnografía interpretativa como un método para el cuidado, propio y de otros, y como un camino para rescatar las vivencias y narrativas de las enfermeras, las cuales se pierden en el día a día.

\section{DESARROLLO}

El cuidado es el fundamento de la ciencia de enfermería y puede ser conceptualizado como todas aquellas acciones que ayudan a preservar y mantener la vida. Se origina en la necesidad de supervivencia del ser humano en circunstancias adversas y, si bien es inherente a la especie, ha estado diferenciado según género, ya que el cuidado de la familia, los alimentos y las necesidades de afecto se han vinculado históricamente a la mujer. En el caso de los hombres, el cuidado se relacionó con los actos que requerían fuerza física y con la consecución de alimentos a través de labores de caza y pesca. Desde su inicio, la enfermería moderna tomó la práctica del cuidado como la base de su disciplina, abordada desde diversos aspectos que incluyen la asistencia, la investigación, la docencia y la gestión ${ }^{3}$.

Watson la definió como una ciencia humana a la que ha denominado "ciencia del cuidado", la cual es un fenómeno único en su interacción recíproca y es parte del mundo que la rodea ${ }^{4}$. En torno al cuidado, manifiesta que es específico, parte de la cultura, los valores y las creencias de las personas, de modo que posiciona la preocupación por los demás como el pilar de la existencia humana: es una forma de vivir y de encontrarse en relación con los otros, estar y convivir en el mundo, característica esencial para toda adaptación ${ }^{5}$. 
La calidad del cuidado no solo está dictada por las intervenciones de la enfermera, sino que es un proceso en conjunto, el cual incluye un vínculo de enfermera-persona, y del paciente-persona, lo cual incluye necesidades y límites, así como la adquisición de destrezas emocionales -como lo son la empatía y la compasión-, el desarrollo personal, profesional y espiritual, mientras se incorpora nuevas experiencias y oportunidades para engrandecer sus propios valores y creencias, de acuerdo con la relación que se establezca con ella misma y su entorno ${ }^{6}$.

Una de las nuevas vías para contribuir al cuidado humanista y centrado en la persona y su familia es la autoetnografía interpretativa, la cual se comprende como el uso de la experiencia y escritura personal para comentar las vivencias y prácticas culturales, denunciar la vulnerabilidad y crear relaciones recíprocas con la audiencia en atención a lograr una respuesta ${ }^{7,8}$. Como ciencia aplicada con un fuerte componente práctico, la enfermería mantiene una continua interacción con el otro, con una comunicación de persona a persona. Al hacer la observación de manera consciente, nos damos cuenta de la conexión con los pacientes, como nos reflejamos en ellos, al compartir experiencias y aprendizajes en que imbricamos nuestro sentir, historias, sensibilidades y cultura reflejándonos en el sufrimiento y alegrías de ellos y los propios.

En relación con lo anterior, Clandinin menciona que las personas crean su vida por medio de historias de quiénes son ellos y los otros e interpretan su pasado en términos de estas historias que se convierten en un portal por el cual las personas ingresan al mundo, interpretan su experiencia y le dan un significado ${ }^{9}$. Cuando valoramos el proceso de enfermería pedimos a los pacientes que nos cuenten "su historia", de esta forma entramos a su mundo, interpretado por ellos y por nosotras, significando o resignificando en las palabras las experiencias. En ese espacio podemos reflexionar respecto de qué nos está sucediendo como enfermeras, como nuestra propia historia nos resuena y hace sentido al escuchar al otro.

Pelias plantea que las metodologías del corazón, dentro de las cuales se encuentra la autoetnografía interpretativa, impulsan al investigador a no refugiarse detrás de la ilusión de la objetividad, por ello, solo pueden ser utilizadas si se está dispuesto a compartir ese lugar donde está el corazón, que nos hace aparecer emocionalmente vulnerable y lingüísticamente evocativo ${ }^{10,11}$. Si llevamos el pensamiento de Pelias a nuestra propia praxis como enfermeras, podemos evocar esos momentos en que nuestros pacientes nos han sentido cercanas y conectadas con el corazón, cuando les hemos dado una palabra de aliento y tomado la mano. En momentos en que hemos sido compasivas con ellos y con nosotras, dejando de lado el escudo que muchas veces usamos, nos hemos mostrado vulnerables siendo cercanas y solidarias en el sufrimiento. Esas instancias que nos mueven y conmueven son las que debemos escribir por medio de la autoetnografía, para transmitir a las futuras generaciones que la tecnología y los aspectos cognitivos, si bien son un apoyo importante, sin el sentir y el corazón, no generan un verdadero cuidado.

La autoetnografía rescata las historias y constituye un enfoque diferente para el estudio de la experiencia humana 7 : para Holman, Adams y Ellis, es el uso de la experiencia y la escritura personal para comentar o criticar las prácticas culturales, para contribuir a las investigaciones existentes, exponer la vulnerabilidad y crear relaciones de reciprocidad con el lector para la consecución de una respuesta; por lo tanto, amplía la mirada al incluir las historias personales que figuran en un contexto social y cultural ${ }^{12}$. 
Para Spry, los textos autoetnográficos revelan las fracturas, puntadas y costuras de la interacción individual con otros en el contexto de la investigación de la experiencia. En la interpretación autoetnografía del texto, los lectores se identifican con fracturas en sus vidas ayudando a la curación en sus propias vidas ${ }^{13}$. Las enfermeras somos muy autocríticas y autoexigentes, continuamente nos evaluamos, hasta ser castigadoras, lo cual produce angustia y ansiedad; por ende, al escribir y reflexionar acerca de la experiencia vivida, volcando nuestras emociones y miedos, podemos contribuir a sanarnos y también a sanar a quienes nos leen, ya que se identifican con nuestros escritos al observar que todos somos vulnerables, podemos sentir dolor y aprender del día a día.

El uso de la propia historia como una forma de entender los procesos vitales propios y de nuestros pacientes, nos permite aprender acerca de nuestras vidas como enfermeras, de manera que es posible crear conocimiento personal, aplicado, construido y expresado por la práctica.

Según Ellis y Bochner, la autoetnografía combina el método de investigación (grafía), cultura (etno) y el self (auto), estos últimos conectados a través del método, por lo que el primer recurso de datos de la investigación es la experiencia personal ${ }^{14}$.

El investigador y lo investigado emergen del proceso autoetnográfico, circunstancia por la que existe un acceso privilegiado a la información que no está disponible para investigadores externos, por ello la conexión entre persona y cultura es fundamental. Para el investigador, el self inserto en el grupo y conectado con la cultura es el punto focal del estudio: según Chang, el componente cultural impulsa a que el autoetnógrafo se mueva más allá de las narrativas, hasta lograr el análisis, interpretación y, a veces, teorización del significado cultural de sus propias experiencias ${ }^{15}$, sin dejar de lado los aspectos éticos que emergen cuando son mencionadas otras personas, manteniendo el anonimato de los involucrados ${ }^{15}$.

Las historias de los otros se convierten en ventanas donde los significados socioculturales de las relaciones del self y los otros son expresados, por ende, Pelias plantea que al escribir es posible ser una voz poética que se acerca a las personas a las cuales cuidamos, de las que escribimos desde el corazón porque el corazón nunca se aleja de lo verdaderamente importante, porque estamos en tiempos de crisis y de desesperanza. Además, entre las características de la autoetnografía interpretativa menciona la posibilidad de dibujar un camino para trabajar nuestros sentimientos, establecer una conexión empática con el otro, a partir de una relación desde el corazón porque los latidos del corazón nos distancian de las máquinas: poder comunicarnos desde el corazón con la conversación, la poesía, la música, la pintura y la danza, respetar nuestra historia, cultura y tradiciones ${ }^{10}$.

De acuerdo con el autor mencionado, dicha metodología cumple con tres funciones: evocativa, reflexiva y la de incorporar la experiencia de ser paciente/ enfermera ${ }^{10}$. La primera permite a los involucrados -sea enfermera, paciente u otro profesional de la salud- recordar hitos en su vida, por ejemplo, cuando estuvieron enfermos o alguna persona significativa para ellos estuvo enferma. En cuanto a la reflexiva, la enfermera piensa la enfermería y puede seleccionar pequeños momentos en su vida y analizarlos sintiendo, tomando conciencia de las emociones asociadas, aromas, palabras, sensaciones táctiles e imágenes. Las anécdotas que compartimos en las jornadas laborales, por ejemplo, la primera vez que quedamos a cargo de un turno o cuando celebramos navidad con nuestros pacientes preferidos o la primera vez que puncionamos un paciente, siendo estudiantes. Todas estas experiencias compartidas, si se sistematizan y escriben, son útiles para las enfermeras que están recién iniciando y también para que pacientes, familias y otros profesionales puedan comprender la labor e importancia de la 
enfermería. Lo anterior también ocurre cuando se utiliza este método en pacientes y sus propias vivencias de enfermedad, lo que permite al expresar sus sentimientos y emociones comenzar con un viaje de sanación y compartir con otros su experiencia, de modo que contribuya a mejorar sustancial del cuidado personal y de pacientes y enfermeras.

Considerando lo mencionado, es posible afirmar que con la autoetnografía interpretativa podemos incorporar la experiencia de ser pacientel enfermera, escribir acerca de la tensión que existe entre lo que pensamos y los que sentimos, o de qué manera se depositan las emociones y pensamientos en nuestro cuerpo.

\section{CONCLUSIONES}

La autoetnografía interpretativa nos obliga a cuestionar constantemente nuestras concepciones sobre los seres humanos, nuestros problemas personales, sociales, físicos, mentales, y espirituales, nuestra posición en relación con el otro. Al utilizar nuestras historias como forma de entender los procesos de vida, propios y de nuestros pacientes, aprendemos de nuestras vidas como enfermeras, comprendiendo la experiencia narrada y de esta forma construimos un camino para formar un conocimiento que es personal, práctico, construido y expresado por la práctica.

Para poder usar esta metodología, la enfermera o enfermero debe conocerse y reconocerse como un ser humano con múltiples posibilidades y potencialidades, imperfecciones y limitaciones $\mathrm{y}$, al mismo tiempo, debe ser capaz de mirarse en el otro, tomar conciencia de que el paciente es un ser humano tan perfecto/imperfecto como es ella.

\section{Declaración de conflicto de intereses}

Las autoras declaran no tener conflicto de intereses. Esta publicación fue autofinanciada.

\section{REFERENCIAS BIBLIOGRÁFICAS}

1. McCurry M. Knowledge for the good of the individual and society. Nursing Philosophy 2010; 11(1): $42-52$

2. Hernández F. La Enfermería en la historia. Un análisis desde la perspectiva profesional. Cultura de los Cuidados. 1997; 1(2): 21-35.

3. Rodríguez-Artavia A. Enfermería ante la deshumanización: una necesidad por ser considerada desde la filosofía de Emmanuel Levinas. Enfermería en Costa Rica. 2011; 32 (1): 37- 42.

4. Guillaumet M, Fargues I, Subirana M, Bros M. Teoría del cuidado humano. Un café con Watson. Metas de Enfermería.2005;8(2):28-32.

5. Watson J. The philosophy and science of caring. Revised and updated edition. Boulder University Press of Colorado; 2008. 


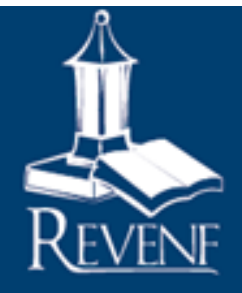

6. Caro S. Enfermería: Integración del cuidado y el amor. Una perspectiva humana. Rev Salud Uninorte. 2009; 25 (1): 172-178.

7. Custer D. Autoethnography as a Transformative Research Method. The Qualitative Report. 2014; 19(37):113.

8. Denzin N. Interpretive Autoethnography. California: SAGE; 2014.

9. Clandinin J. Engaging in Narrative Inquiry (Developing Qualitative Inquiry). California: SAGE; 2013.

10. Pelias R. A methodology of the heart; evoking academic and daily life. Washington: AltaMira Press; 2004.

11. Ramírez-Pereira M, Lobos M, Zapata-Sepúlveda P. Interpretive Autoethnography as a Way of Social Transformation in Academic Teaching and Learning Spaces in Chile. Cultural Studies Critical Methodologies. 2016; 18: 1-8.

12. Adams T, Holman S, Ellis C. Autoethnography Understanding Qualitative Research. Estados Unidos: Oxford University Press; 2015.

13. Spry T. Performing Autoethnography: An Embodied Methodological Praxis. Qualitative Inquiry.2001; 7(6): 706-732.

14. Ellis C, Bochner A.(2002) Ethnographically Speaking. Walnut Creek, CA: Altamira Press; 2002.

15. Chang, H. Autoethnography in Health Research: Growing Pains? Qualitative Health Research. 2016; 26(4) $443-451$. 